\title{
Structure and electronic structure of quasicrystal and approximant surfaces: a photoemission study
}

\author{
D. Naumović
}

Département de Physique, Université de Fribourg, Pérolles, CH-1700 Fribourg, Switzerland

\begin{abstract}
The structure and electronic structure of different high-symmetry surfaces of either quasicrystalline or approximant $\mathrm{Al}-\mathrm{Pd}-\mathrm{Mn}$ were studied by means of photoemission-based techniques such as X-ray photoelectron diffraction (XPD) and ultraviolet photoelectron spectroscopy. We find that the twofold (2f), $3 f$ and $5 f$ surfaces of icosahedral Al-Pd-Mn exhibit all the symmetry elements of the icosahedral non-crystallographic group. These XPD experiments can be modeled by single-scattering cluster calculations.

The bulk-terminated icosahedral or approximant surfaces are recovered after ion sputtering followed by annealing at $T \approx 500-600{ }^{\circ} \mathrm{C}$. A wealth of ordered surface phases (with different compositions) are found after sputtering and depending on the annealing temperature as, for example, a crystalline bcc multitwinned phase (for $T<400{ }^{\circ} \mathrm{C}$ ) or a stable decagonal quasicrystalline surface (for $T>650{ }^{\circ} \mathrm{C}$ ).

The icosahedral surfaces are characterised by a lowering of the density of states close to the Fermi edge, compatible with the opening of a pseudogap, as expected for a quasicrystal. The crystalline overlayers are characterised by a sharp Fermi edge, while the approximant and decagonal quasicrystalline surfaces also have a lowered density of states.
\end{abstract}

Keywords: Quasicrystal; Approximant; Decagonal; Icosahedral; Al-Pd-Mn; X-ray photoelectron diffraction (XPD); Ultraviolet photoelectron spectroscopy (UPS)

* Tel.: +41-26-300-9089; fax: +41-26-300-9747.

E-mail address: dusanka.naumovic@unifr.ch (D. Naumović).

0079-6816

doi:10.1016/j.progsurf.2004.05.004 


\section{Introduction}

Quasicrystals (QC), besides their fascinating structure infringing the well-established rules of conventional crystallography [1], exhibit most remarkable electronic and mechanical properties [2]. More practically, quasicrystal coatings start to be used for real industrial applications, and this is due to the unusual low friction, low adhesion and increased hardness characteristics of their surfaces. It is therefore of prime interest to study these surfaces on an atomic scale using surface science methods (for a review, see this volume). Crucial questions concerning the stability of the quasicrystalline surfaces and their properties under different treatments as annealing or oxidation are specifically addressed to surface scientists.

Standard crystallographic methods operating in reciprocal space, such as neutron, $\mathrm{X}$-ray or electron diffraction, have already provided nearly coherent models for quasicrystals, but detailed atomic positions and the chemical occupation on different sites are still unclear. Scanning tunneling microscopy [3], secondary-electron imaging [4] and low-energy electron diffraction (LEED) [5] are surface techniques highly sensitive to local order. Note that LEED is again a diffraction technique operating in reciprocal space. None of them, however, is chemically selective. In the present study we will show results from a photoemission-based method, X-ray photoelectron diffraction (XPD) [6], which is chemically selective and sensitive, providing at the same time local, site-specific, real-space information of the near-surface region. In combination with single-scattering cluster calculations (SSC) [6], XPD can efficiently contribute to modelling quasicrystal surfaces. 
On the other hand, separate measurements on the electronic structure have been carried out with valence-band ultraviolet-photoemission spectroscopy (UPS). Since specific heat measurements [7] indicated a significant reduction of the density of states (DOS) at the Fermi level $E_{\mathrm{F}}$, the debate was whether such a reduction of DOS (or a pseudogap) is observed in UPS or whether the very surface is metallic. Recently it was found that the apparent metallicity at the surface is decreasing with increasing sampling depth [8]. Finally, despite the lack of periodicity, a bandlike behaviour was observed in the electronic structure $[9,10]$.

So, here, we exploited three aspects of photoemission: in the soft X-ray regime, XPS for evaluating the composition and full-hemispherical XPD for revealing the structure, and, in the ultraviolet (UV) regime, UPS for testing the electronic structure. As an additional technique, we used also LEED for checking the structure. This set of in-situ tools enables to investigate the structure and electronic properties in parallel and on the same surface.

Most of our work, as summarised in Fig. 1, was devoted to icosahedral $i$ $\mathrm{Al}_{70} \mathrm{Pd}_{20} \mathrm{Mn}_{10}$ quasicrystals, which were the first stable icosahedral quasicrystals discovered [11] and available as high-quality large monograin. We prepared clean surfaces of different faces $(5 f, 3 f$ and $2 f)$ by ion sputtering followed by annealing. An icosahedral bulk-terminated surface [12], characterised by a lowered DOS close to $E_{\mathrm{F}}$ was obtained after annealing at temperatures between roughly 450 and $650{ }^{\circ} \mathrm{C}$ $[13,14]$ (Section 3). XPD patterns taken on this surface were used for modelling with the help of SSC calculations [15] (Section 4). Interestingly, we found also ordered,

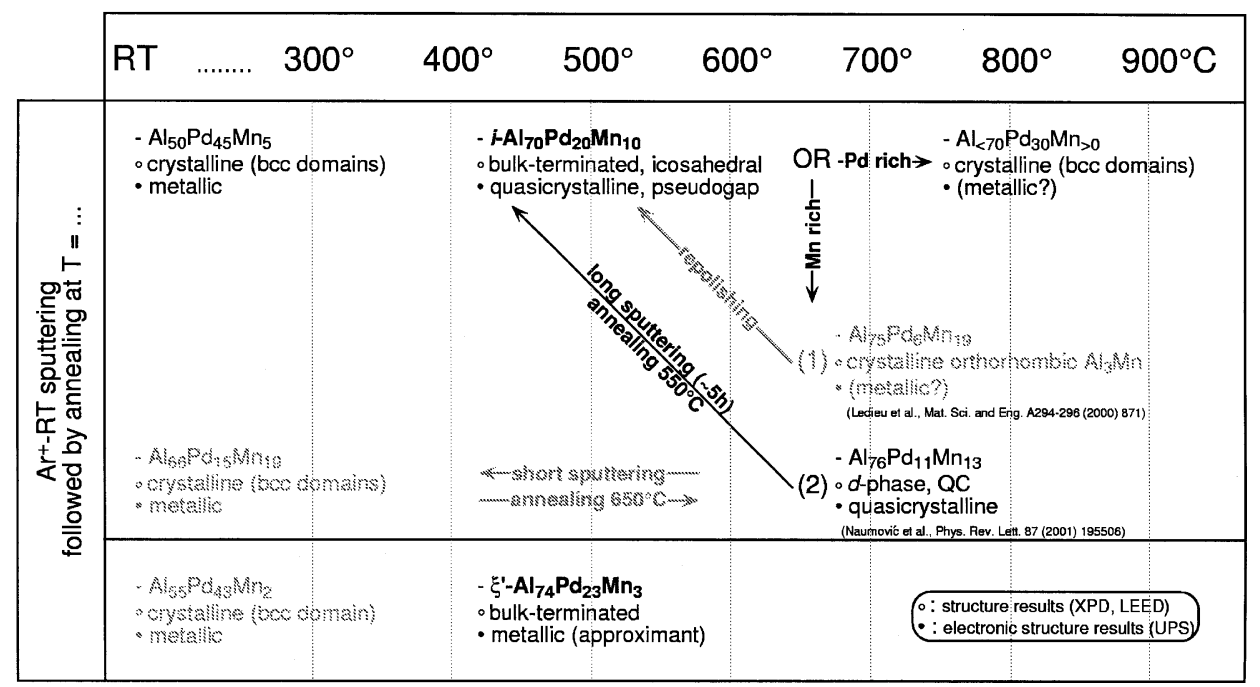

Fig. 1. Overview of phases at surfaces of $i-\mathrm{Al}-\mathrm{Pd}-\mathrm{Mn}$ quasicrystal and $\xi^{\prime}-\mathrm{Al}-\mathrm{Pd}-\mathrm{Mn}$ approximant obtained after ion sputtering followed by annealing at different temperatures. The bulk-terminated surfaces are indicated in bold. The results indicated in grey are not discussed in the paper or not measured by us. Open circles indicate results from structural investigations (XPD, LEED) and black circles results concerning electronic structure (UPS). 
but crystalline and twinned, surfaces after sputtering only or after sputtering followed by annealing below $400{ }^{\circ} \mathrm{C}[12,14,15]$ (Section 5). When annealing at temperatures above $650{ }^{\circ} \mathrm{C}$, we observed either Pd enrichment $[12,16]$ and similar crystalline reconstructions as for low temperature annealings or Mn enrichment (Section 6). In the latter case we observed the formation of a stable decagonal $d$-Al$\mathrm{Pd}-\mathrm{Mn}$ [17] quasicrystal overlayer [18], what is exceptional as single-grain samples of this phase could not be produced up to now, and are only possible in this system because $\mathrm{Al}-\mathrm{Pd}-\mathrm{Mn}$ has the only known phase diagram containing two stable QC phases the $i$ - and $d$-phase [19]. This overlayer was thick enough (200-500 A) to permit a complete characterisation as well as sputtering/annealing experiments. Note that also different reconstructions of $\mathrm{Mn}$ rich overlayers were observed by others $[20,21]$. The high-temperature phases will be compared to data obtained from $\xi^{\prime}-\mathrm{Al}-$ $\mathrm{Pd}-\mathrm{Mn}$ approximant sample (Section 6), which is a crystalline periodic phase with quasicrystal-like ordering within its big unit cells [22].

Finally, we performed also oxidation experiments and observed that $\mathrm{Al}$ in the $i$ $\mathrm{Al}-\mathrm{Pd}-\mathrm{Mn}$ surface reacts less that in the crystalline $\mathrm{Al}-\mathrm{Pd}-\mathrm{Mn}$ environment, but more than in $\mathrm{Al}\left(\begin{array}{ll}1 & 1\end{array}\right)$ [23]. But oxidation destroys ordering of the surfaces; thus these data will not be discussed further.

\section{Experiment and calculations}

Photoemission has a long-standing tradition in surface analysis. A typical experimental setting for a photoemission experiment is shown in Fig. 2a, consisting here of a fixed electron analyser and an X-ray source. Operating in the soft X-ray regime, XPS is used to study the chemical composition of surfaces. As the photon is absorbed via the photoelectric effect, photoelectrons from different core levels are emitted and arrive at the detector with different kinetic energies; in the experiment, intense core-level peaks appear in the energy spectrum as the energy is scanned. Distinct species are therefore easily distinguishable. The experiments are either done in an angle-integrated or angle-resolved mode. Integrated-mode experiments study concentrations of chemical species in XPS by comparing the intensity of core-level peaks of two (or more) chemical species present in the studied surface.

Angle-resolved photoemission or XPD on the other hand is able to study atomic positions near the surface in real space [6]. The variation of intensity of monoenergetic photoelectrons, originated from a selected atomic species, is measured at different angles above the surface. Practically, azimuthal intensity scans, obtained by rotating the sample around its surface normal ( $\Phi$ angle), are measured starting from grazing up to normal emission ( $\Theta$ angle) (Fig. 2a). The intensities are then stereographically projected as a grey-scale map, as shown for $\mathrm{Cu}(001)$ (Fig. 2b). The centre and the outer ring correspond to normal and grazing emission, respectively; one full map contains more than 5000 angular settings. For kinetic energies above about $500 \mathrm{eV}$, photoelectrons are focused along dense atomic rows or planes of a given structure creating intensity maxima along these directions (Fig. 2c). So the mapping of the core-level electron intensity over the hemisphere above the sample 

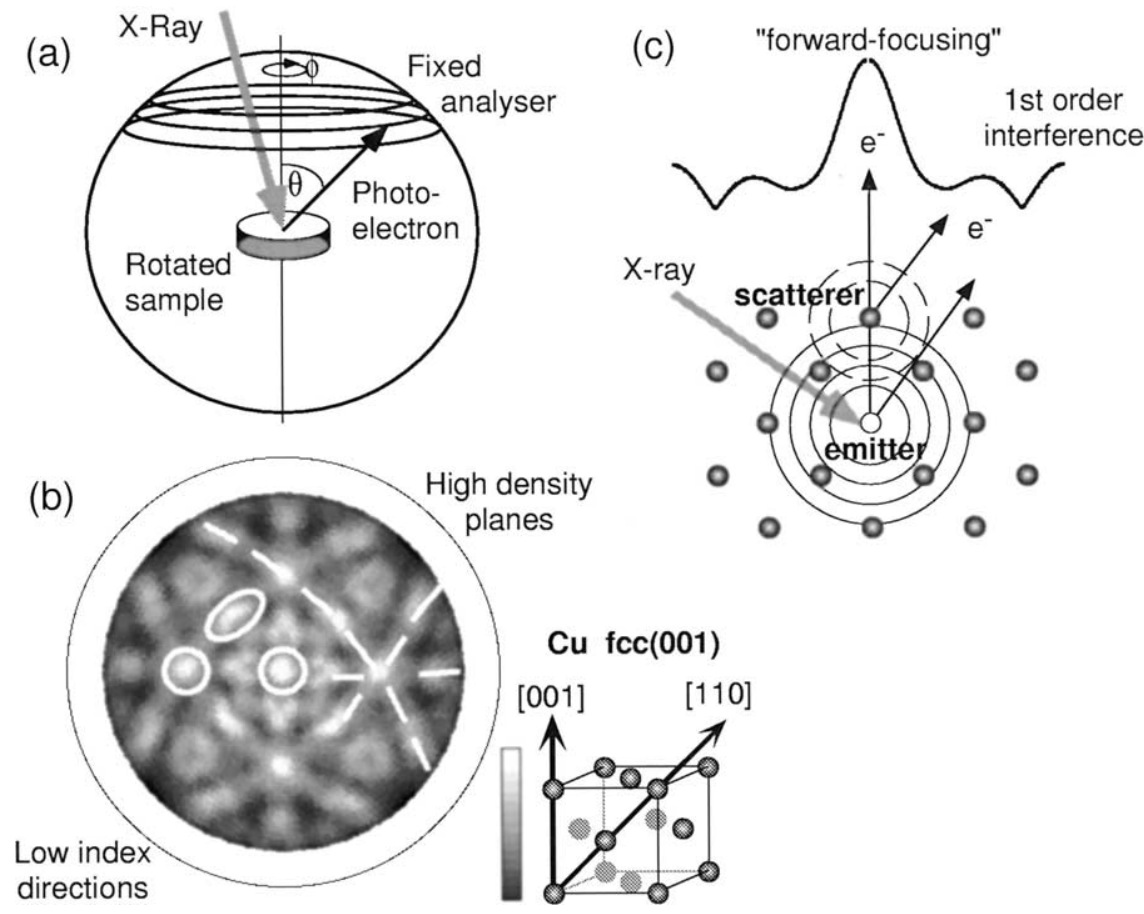

Fig. 2. (a) Experimental set-up with a fixed X-ray (MgK $\alpha$ and $\mathrm{SiK} \alpha$ ) (or UV source (He I) for UPS) and a fixed electron-energy analyser. For XPD, the sample is rotated around two axes. Sample rotation and data acquisition are automated. The photoelectron intensities recorded at each angular position for a given kinetic energy are stereographically projected as shown in (b) for $\mathrm{Cu}(001)\left(\mathrm{Cu} 2 \mathrm{p}_{3 / 2}, 807 \mathrm{eV}\right)$. Forwardfocusing maxima are present for emission along dense atomic rows in the normal [0 01 1] direction and in the $45^{\circ}$ off-normal [ 110 ll-like directions (white circles). High-density planes are also visible (dashed lines). (c) For kinetic energies of photoemitted electrons above about $500 \mathrm{eV}$, the scattered intensity is maximal along the bond axis. First-order interference fringes contain bond-length information.

surface results in a projection of the atom-atom directions, starting from the core level of the emitting chemical species, which appear as intense spots (in white) in the map [24,25]. Besides this forward-focusing effect, changes of intensity are also produced by constructive or destructive interferences of the outgoing electron wave with its scattered wave as a function of angle. So, the diffractogram obtained from a particular emitter gives a very specific and unique view of its local real-space environment. In short, XPD is chemically selective and sensitive, providing at the same time averaged local and site-specific, real-space information of the near-surface region. Therefore, XPD is particularly suitable to probe surface reconstructions and phase transitions occurring at surfaces of monocrystals or monograin quasicrystals.

The XPD process can be simulated with scattering calculations [6]. In our case we used single-scattering cluster calculations (a detailed description of the parameters is given in [25]). The main purpose of these simulations is to refine model clusters, i.e., atomic positions until best agreement with experiment is yielded. With SSC, 
calculations with a great number of emitters using large clusters can be performed in a reasonable time, enabling testing of many different configurations.

Photoemission performed in the UV regime (UPS) enables measuring the density of states close to the Fermi edge, and thus provides information on the electronic structure.

The experiments were performed on monogram samples of the $i-\mathrm{Al}-\mathrm{Pd}-\mathrm{Mn}$ quasicrystal and $\xi^{\prime}-\mathrm{Al}-\mathrm{Pd}-\mathrm{Mn}$ approximant (grown using the Czochralsky or Bridgman method at CECM-CNRS (Vitry-sur-Seine, France), Ames Laboratory (USA) or Forschungszentrum Jülich (Germany)) cut perpendicularly to a highsymmetry axis $(5 f, 3 f, 2 f$ or pseudo-10f) and polished. Surface preparation and photoemission experiments were performed in a VG ESCALAB Mark II UHV spectrometer with a base pressure in the $10^{-11}$ mbar range and equipped with a LEED apparatus, a twin X-ray anode $(\mathrm{MgK} \alpha(h v=1253.6 \mathrm{eV})$ and $\mathrm{SiK} \alpha(h v=1740$ $\mathrm{eV})$ ), a monochromatised UV source (He I, $h v=21.2 \mathrm{eV}$ ) and a two-axis goniometer. Clean $i$-Al-Pd-Mn surfaces were prepared in situ by repeated cycles of $\mathrm{Ar}^{+}$ sputtering and annealing. After this treatment LEED indicated a well-ordered surface (not shown). All photoemission measurements were performed at room temperature. The probed depth is approximately $20-50 \AA$, depending on the energy of photoelectrons. Surface contaminations and concentrations were checked with XPS. The same core-level photoemission lines, Al 2s, Pd 3d and Mn 2p, were used to evaluate the composition (intensities weighted with the corresponding cross-sections $\left.\left(I_{0} / \sigma\right)\right)^{1}$ and the XPD pattern, ensuring, thus, that the measured composition and structure are generated from the same depth.

\section{Icosahedral quasicrystalline surfaces}

In this section (Figs. 3-6), we present the clean quasicrystalline surfaces of $i$-Al$\mathrm{Pd}-\mathrm{Mn}$, i.e., the bulk-terminated surfaces. The first questions to be solved were how to prepare such surfaces and how to check whether the prepared surfaces have the expected termination. We proceeded by ion sputtering followed by annealing cycles. The annealing temperature is situated between roughly 450 and $650{ }^{\circ} \mathrm{C}$, but ideally in the $500{ }^{\circ} \mathrm{C}$ range. As composition changes are induced by preferential sputtering of the lightest elements and by thermal diffusion, the duration of the annealing can also considerably influence the result. For lower or higher temperatures and depending on the annealing duration, a wealth of reconstructions are obtained; most of them are crystalline and present interesting structural coincidences with the quasicrystal bulk, but can also be quasicrystalline with a different structure (Fig. 1 and Sections 5 and 6).

We characterised the quasicrystalline surface using four different techniques as follows. The composition is measured with XPS and is roughly $\mathrm{Al}_{70} \mathrm{Pd}_{25} \mathrm{Mn}_{5} .{ }^{1} \mathrm{The}$

\footnotetext{
${ }^{1}$ Note that XPS concentrations cannot be directly compared with a true stoichiometry because it is based only on intensity ratios including core-level cross-sections.
} 


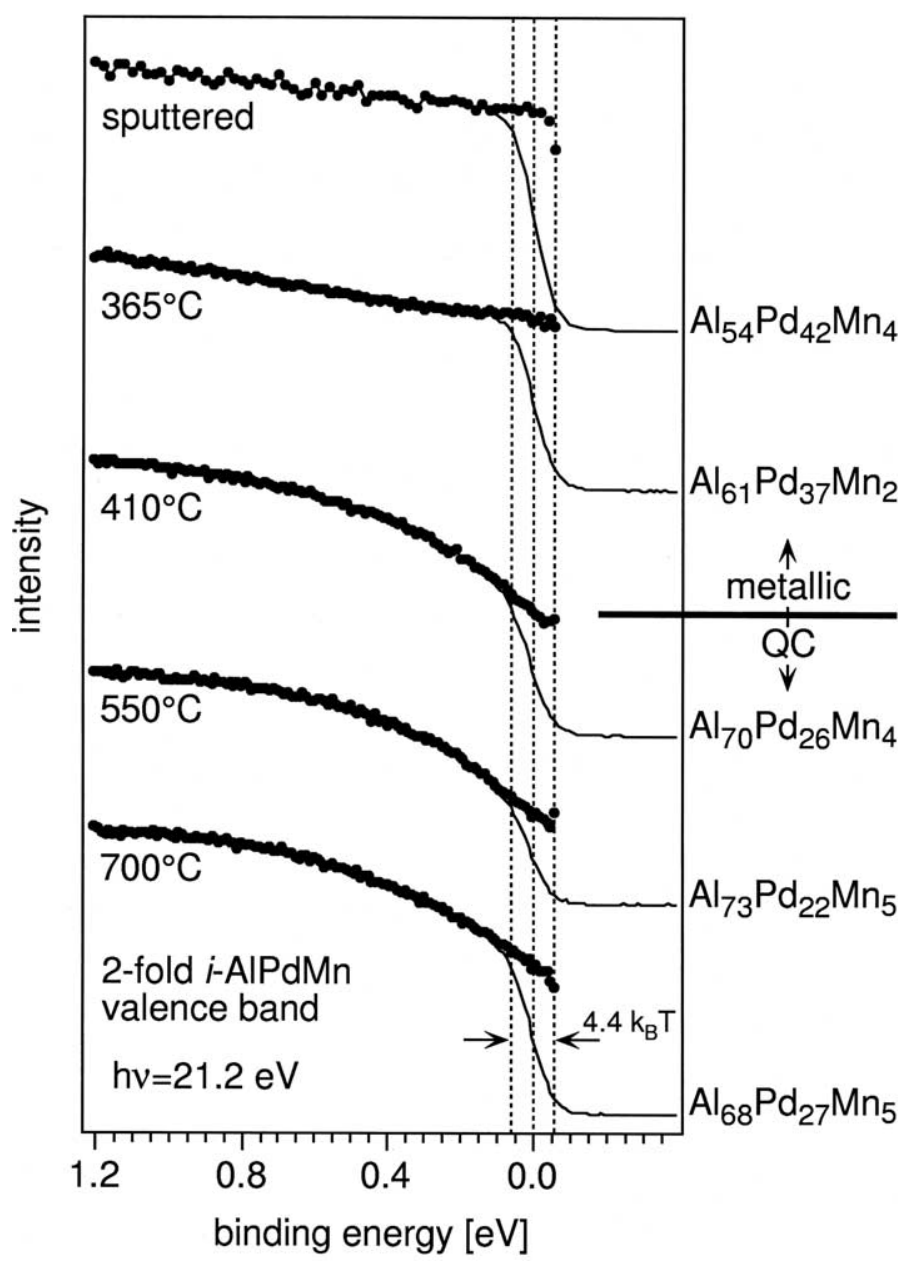

Fig. 3. Valence-band spectra displaying the region near the Fermi edge, taken with monochromatised He I radiation at room temperature, of $2 f i-\mathrm{Al}-\mathrm{Pd}-\mathrm{Mn}$ surfaces treated by sputtering followed by annealing at labeled temperature. Spectra (thin black lines) are divided by the Fermi-Dirac distribution in order to extrapolate DOS at the Fermi cut-off. DOS is linear as for metals for $T_{\text {anneal }}<400{ }^{\circ} \mathrm{C}$ and lowered $>400{ }^{\circ} \mathrm{C}$ as expected from quasicrystals. XPS compositions are indicated for each spectrum.

structure, as checked by LEED (not shown) and XPD, exhibits the typical features of icosahedral symmetry elements. Finally, UPS, which is extremely sensitive to slight disordering of the surface (such as a 1-min sputtering [26]), shows a distinct suppression of spectral weight in the DOS close to $E_{\mathrm{F}}$.

Since the electronic structure fingerprints as measured by UPS are particularly efficient in characterising the prepared surfaces, we show (as an example) valenceband spectra of $2 f i$-Al-Pd-Mn surfaces after sputtering followed by annealing at labeled temperatures in Fig. 3 [13]. The fine black lines represent the measured 

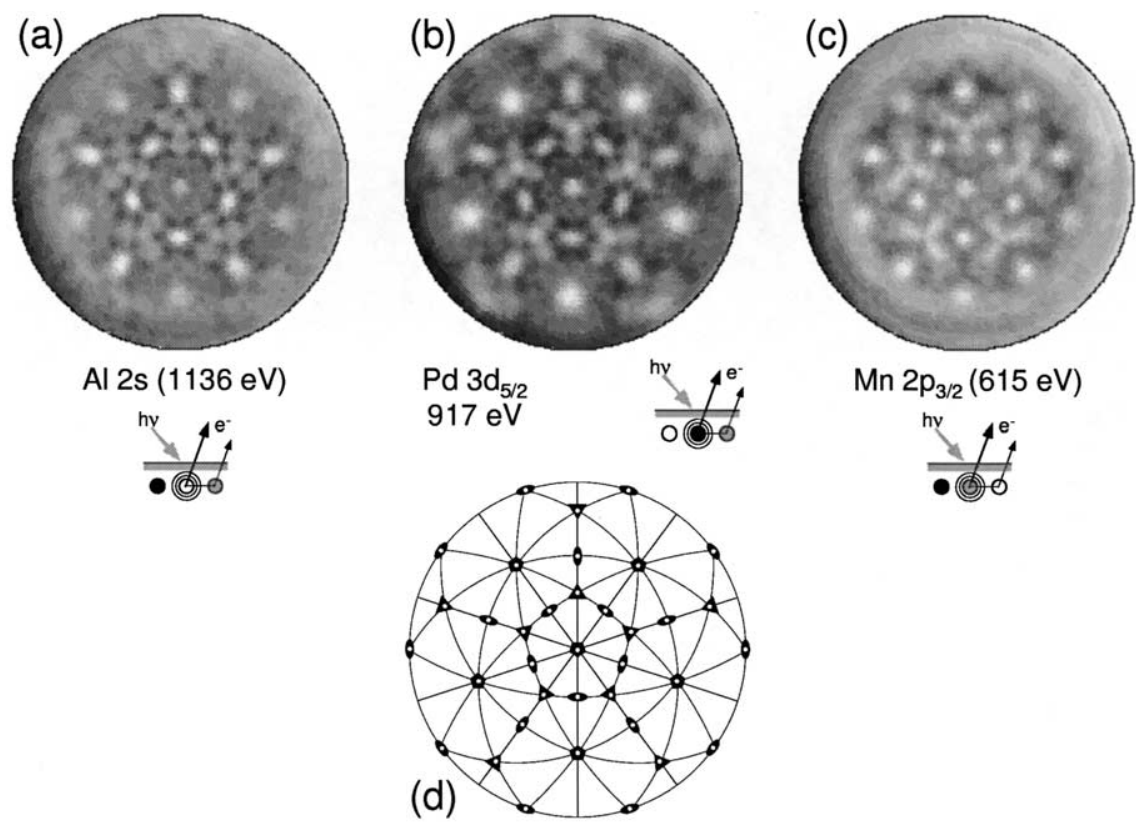

$\mathrm{Mn} \mathrm{2p_{3/2 }}(615 \mathrm{eV})$

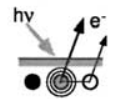

Fig. 4. Experimental XPD patterns of a quasicrystalline surface of $5 f i$-Al-Pd-Mn for emission from (a) $\mathrm{Al} 2 \mathrm{~s}$, (b) $\mathrm{Pd} 3 \mathrm{~d}_{5 / 2}$ and (c) $\mathrm{Mn} 2 \mathrm{p}_{3 / 2}$ measured with an $\mathrm{MgK} \alpha(1253.6 \mathrm{eV}) \mathrm{X}$-ray source; emitting atoms are indicated in white, black and grey, respectively. (d) shows the stereographic projection of the icosahedral symmetry elements with axes of $2 f$ (ellipses), $3 f$ (triangles) and $5 f$ (pentagons) symmetry.

spectra. The black dots have been obtained by normalizing the spectra with the Fermi-Dirac distribution function, therefore removing the sharp cutoff and creating a spectral function representing the DOS near $E_{\mathrm{F}}$ within a range of approximately 4.4k $k_{\mathrm{B}} T$, where $k_{\mathrm{B}}$ is the Boltzmann constant [8]. $4.4 k_{\mathrm{B}} T$ represents the region where the Fermi-Dirac distribution function takes values between $90 \%$ and $10 \%$. First we notice the characteristic changes of the shape of the Fermi edge. Whereas the two topmost spectra show linear behaviour over basically the complete range of energies, what is characteristic of metallic surfaces, the surfaces annealed above $400{ }^{\circ} \mathrm{C}$ exhibit drastically collapsing intensities for energies approaching $E_{\mathrm{F}}$. Such a distinct decrease of DOS near $E_{\mathrm{F}}$ may be interpreted as a pseudogap and is attributed to QC surfaces. The behaviour of the DOS near $E_{\mathrm{F}}$ is identical for the $5 f[14]$ and $3 f$ surfaces. It is also interesting to note that the spectral weight close to $E_{\mathrm{F}}$ is essentially attributed to Mn 3d states [27].

Figs. 4-6(a)-(c) show experimental XPD intensity maps of different quasicrystalline icosahedral surfaces of $i$-Al-Pd-Mn. In Fig. 4(a)-(c), the XPD diffractograms are presented for the three elements of a $5 f i$-Al-Pd-Mn surface using $\mathrm{MgK} \alpha$ radiation $(1253.6 \mathrm{eV})$. By choosing a particular emission line or emitting atom (Pd, $\mathrm{Al}$ or $\mathrm{Mn}$ ), we probe this specific local real-space environment around the selected emitting atoms. An overall inspection of the maps shows a $5 f$ symmetry for all three elements. 

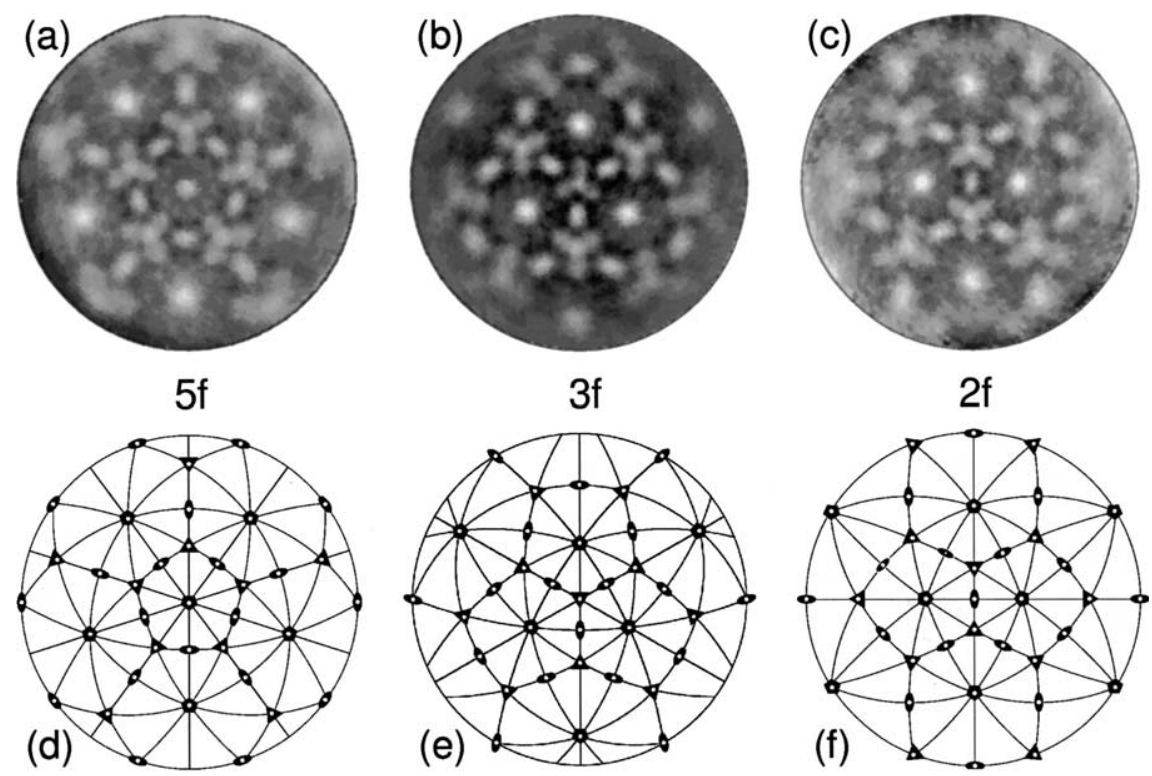

Fig. 5. Experimental XPD patterns of quasicrystalline surfaces of $i$-Al-Pd-Mn cut perpendicularly to (a) a $5 f$ axis, (b) a $3 f$ axis and (c) a $2 f$ axis, taken at the same kinetic energy (917 eV) for $\mathrm{Pd} 3 \mathrm{~d}_{5 / 2}$ emission (with $\operatorname{MgK} \alpha(1253.6 \mathrm{eV}))$. (d)-(f) shows the corresponding stereographic projection of the icosahedral symmetry elements with axes of $2 f$ (ellipses), $3 f$ (triangles) and $5 f$ (pentagons) symmetry.

Clear and well-defined forward-focusing maxima are observed as well as a considerable amount of fine structure which is due to interference. For all three elements $\left(\mathrm{Al} 2 \mathrm{~s}(1136 \mathrm{eV}), \operatorname{Pd} 3 \mathrm{~d}_{5 / 2}(917 \mathrm{eV})\right.$ and $\left.\mathrm{Mn} 2 \mathrm{p}_{3 / 2}(615 \mathrm{eV})\right)$, the kinetic energy is in the forward-focusing regime. Comparing the patterns with the stereographic projection of the icosahedral symmetry elements (Fig. 4d) we can clearly identify axes of $5 f, 3 f$ and $2 f$ symmetry. This is best seen for Pd where the $5 f$ axes have the shape of a $5 f$ star, the $3 f$ axes look like trefoils and the $2 f$ axes are elongated (Fig. $4 \mathrm{~b}$ ). Therefore, we conclude $\mathrm{Al}, \mathrm{Pd}$ and $\mathrm{Mn}$ are all sitting in an environment of icosahedral symmetry.

Fig. 5 presents XPD maps of Pd emission taken from three samples of different orientations (cut perpendicularly to a $5 f, 3 f$ and $2 f$ axis) and the corresponding stereographic projections. Here, again, we can clearly identify axes of $5 f, 3 f$ and $2 f$ symmetry. But the normal emission, in the centre of the pattern, which corresponds to $5 f$ axis in $5 f i$-Al-Pd-Mn (Figs. 4 and $5 \mathrm{a}$ ), is replaced by a $3 f$ axis (Fig. $5 \mathrm{~b}$ ) and a $2 f$ axis (Fig. 5c) at the normal in the $3 f i$-Al-Pd-Mn and $2 f i$-Al-Pd-Mn samples, respectively. Intensity maxima shaped as stars, trefoils or ellipses can be found in the latter two maps as discussed for the $5 f i$-Al-Pd-Mn case. Therefore, we can conclude that the surfaces of all the three orientations are icosahedral, terminated as the bulk.

Fig. 6(a)-(c) display XPD diffractograms for the three elements of a $2 f i$-Al-Pd$\mathrm{Mn}$ surface using $\mathrm{SiK} \alpha$ radiation $(1740 \mathrm{eV})$. The reason for changing the radiation 

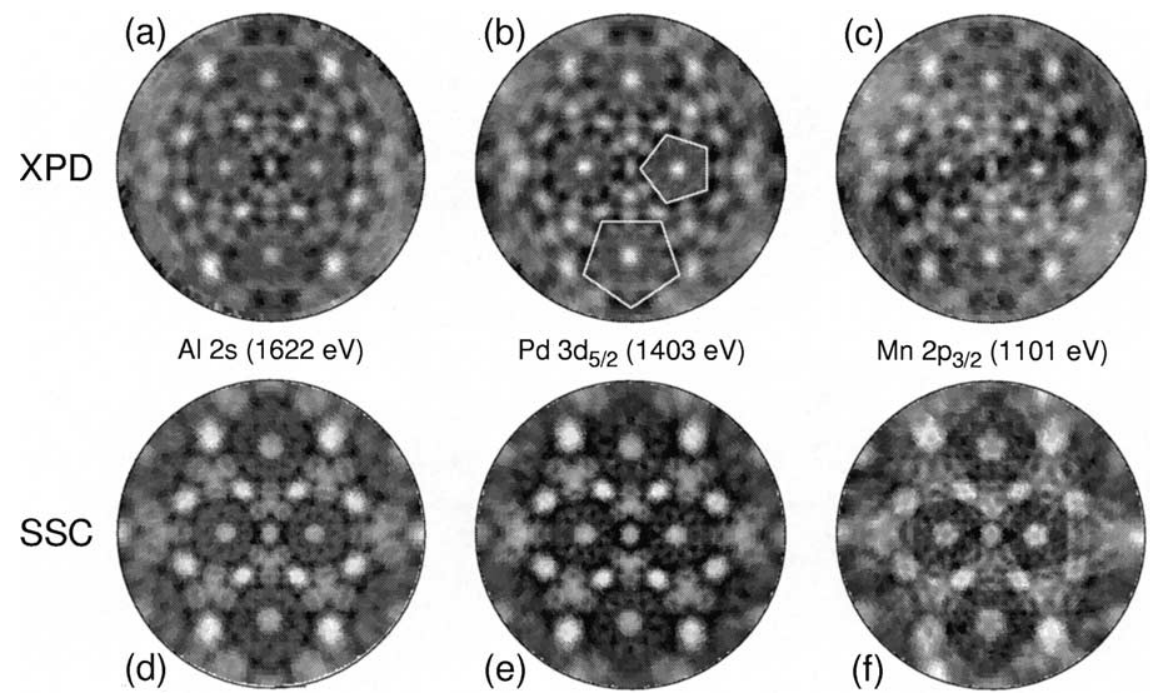

Fig. 6. Experimental XPD patterns of a quasicrystalline surface of $2 f i$-Al-Pd-Mn for emission from (a) Al2s, (b) $\mathrm{Pd} 3 \mathrm{~d}_{5 / 2}$ and (c) Mn 2 $\mathrm{p}_{3 / 2}$ measured with a SiK $\alpha(1740 \mathrm{eV}) \mathrm{X}$-ray source; the white pentagons in (b) emphasize the shape and the orientation of features due to interferences appearing around $5 f$ axes. Corresponding simulated patterns with SSC using a spherical cluster $(r \leqslant 16 \AA)$ of 1111 atomic positions with (d) $784 \mathrm{Al}$ emitters, (e) $228 \mathrm{Pd}$ emitters and (f) $99 \mathrm{Mn}$ emitters.

source is to reach a stronger forward-focusing regime where the influence of interference is diminished with respect to the direct forward-focusing induced information. All the maps have a $2 f$ symmetry and can be compared with the corresponding stereographic projection of the icosahedral symmetry elements (Fig. 5f).

Furthermore, we observe that the $2 f$ axes are interconnected in both Al patterns taken at different energies from a $5 f i$-Al-Pd-Mn (Fig. $4 \mathrm{a}$; for the stereographic projection with the $2 f$ axes interconnected, see below (Fig. $8 b)$ ) and $2 f i$-Al-Pd-Mn substrate (Fig. 6a). This indicates that the $2 f$ axes lie on atomically dense planes. The Pd signals (Figs. 4b and 6b) do not show such connecting lines. In general, highdensity planes are most easily recognized at higher kinetic energies but looking at Fig. $2 \mathrm{~b}$ we find that high-density planes are already visible at $807 \mathrm{eV}$ for $\mathrm{Cu}$. So, probably this has to do with different atomic sites taken by the three elements and it may indicate that $\mathrm{Pd}$ is not located within these high-density planes seen around $\mathrm{Al}$. For Mn (Figs. 4c and 6c) the situation is less clear because its signal arrives at much lower kinetic energy (for $\mathrm{MgK} \alpha$ radiation) and the statistics is weaker due to the smaller concentration. Nevertheless high-density planes do not seem to be present. Furthermore, note that the Mn XPD map taken at $615 \mathrm{eV}$ (where the probed depth is also smaller) (Fig. 4c) looks different than the XPD maps of $\mathrm{Al}$ and Pd (Fig. 4a and b); it exhibits, for instance, much bigger trefoil patterns; while such differences are not observed in the data set taken at higher energy (Fig. 6(a)-(c)), i.e., in a stronger forward-focusing regime. So we can conclude that the interconnection of $2 f$ axes 
observed in Al XPD maps is a real structural feature, linked to a specific atomic environment, whereas the apparent differences of the Mn XPD map taken at a low kinetic energy is purely an interference effect.

\section{Modelling}

The determination of the atomic arrangement in quasicrystals is far from being straightforward and simple. Boudard et al. [28] determined the structure of $i$-Al-Pd$\mathrm{Mn}$ from X-ray and neutron diffraction data. Owing to the large difference between $\mathrm{X}$-ray and neutron scattering factors, information is gained on the atomic position of the three elements. The resulting 3D structure contains icosahedral clusters similar to the external shell of the Mackay icosahedron (for description, see below) and an information on the chemical decoration. Then different models were proposed in order to reproduce the structure obtained from experiment [29-31]. Besides the pseudo-Mackay icosahedron ("pseudo" because the inner shell differs from the "true" Mackay icosahedron), the Bergman dodecahedron was found to appear in the structure. In one model, the pseudo-Mackay icosahedron [28,29] was used as the key cluster to explain how the structure is generated; it consists of a hierarchical packing of pseudo-Mackay icosahedra. Other models are based on the Bergman dodecahedron [30,31]. In one of them, the space is filled with two types of tiles, an oblate and a prolate rhombohedron each decorated with Bergman dodecahedra [31].

In the previous section, we presented experimental XPD data, which show all the fingerprints of an icosahedral geometry. But in order to model the specific arrangement of atoms close to the surface, a comparison with SSC simulations using atomic position proposed by bulk models is necessary. First, such a comparison can check if the probed surface (up to $50 \AA$ ) is in agreement with the bulk arrangement. On the other side, since we have the indication from electronic structure measurements (UPS) that the surface is quasicrystalline, we can verify the validity of the model. Furthermore, as XPD is sensitive to the local atomic structure, some details of the bulk structure may be elucidated, which could not easily be obtained from the X-ray and neutron diffraction data [28] as, for instance, the geometry of the inner shell of the pseudo-Mackay icosahedra. Finally, SSC model calculations can also provide further information on the chemical occupation of different sites.

In Fig. 6, a set of experimental XPD maps ((a)-(c) described in the previous section) and SSC simulations (d)-(f) for $\mathrm{Al}, \mathrm{Pd}$ and $\mathrm{Mn}$ emission of a $2 f i-\mathrm{Al}-\mathrm{Pd}-\mathrm{Mn}$ surface are shown. For the calculations we used a cluster based on a data file of the Elser model [31]. ${ }^{2}$ It consisted of a sphere $(r \leqslant 16 \AA)$ of 1111 atomic positions

\footnotetext{
${ }^{2}$ These data files of the Elser model [31] were produced by W. Liebermeister, Institut für Theoretische Physik, Tübingen, Germany.
} 
centred on a pseudo-Mackay icosahedron. To each atomic position is attributed a single chemical species: thus, $784 \mathrm{Al}$ emitters simulated the $\mathrm{Al}$ diffractogram, while for Pd and Mn, we had 228 and 99 emitters, respectively. We observe that the simulated maps show intensity maxima at the same positions as the experimental maps. Similar features at $2 f, 3 f$ and $5 f$ axes (Fig. $5 \mathrm{f}$ ), such as trefoils at $3 f$ axes, appear. The agreement for $\mathrm{Mn}$ is less good, but $\mathrm{Mn}$ has the lowest concentration, so the number of emitters was not sufficient to fully reproduce all the features. The pentagonal grey features (marked with a white pentagon in Fig. 6b), which are due to interferences, appear around $5 f$ axes with the same orientation in simulation as in experiment. It is important to note that such an agreement could not be achieved with smaller clusters, and that the same agreement is obtained for arbitrary centred cluster within the data file. It appears also that the interconnection of $2 f$ axes (Fig. 6a) observed in the experimental $\mathrm{Al}$ diffractogram is also present in the simulated map, confirming the presence of $\mathrm{Al}$ in these atomically dense planes; while, as expected, this is not observed in the Pd calculated pattern. So, experimental data are consistent with the bulk model. Finally, the questions concerning the terminating plane of the quasicrystal surface and whether there is a reconstruction or relaxation within the two or three terminating layers, was answered by dynamical LEED, a very surface sensitive technique [32].

As we mentioned above, the position of atoms within the inner shell of the pseudo-Mackay cluster could not be directly extracted from X-ray and neutron diffraction data [28], even if proposed later in models [29]. Fig. 7a shows the pseudoMackay and Mackay icosahedra (or clusters). The two outer shells of a Mackay cluster consist of an outer icosidodecahedron with 30 atoms on $2 f$ axes, an intermediate icosahedron with 12 atoms occupying positions on $5 f$ axes. The inner shell of the true Mackay cluster is again an icosahedron; while in the pseudo-Mackay cluster, it is replaced by an inner, partially occupied dodecahedron with 8 of 20 possible positions on the $3 f$ axes. Both clusters have a central atom in addition. We performed simulations with the $\mathrm{Al} 2 \mathrm{~s}$ emission since $\mathrm{Al}$ has been proposed to sit on all the different shells of the pseudo-Mackay cluster [28,29]. Fig. $7 \mathrm{~b}$ and c show SSC simulations using a pseudo-Mackay cluster and a Mackay cluster, respectively. By comparing both with the experimental pattern (Fig. 7d), we observe much better agreement for the pseudo-Mackay cluster, especially in the $3 f$ and $2 f$ directions (see Fig. 4d). Note that the Mackay icosahedron appears in the structure of the $i-\mathrm{Al}-\mathrm{Mn}-\mathrm{Si}$ quasicrystal. Here, it is interesting to remark that a cluster as small as a pseudo-Mackay icosahedron (51 atoms) is sufficient to reproduce the main features of the $i$-Al-Pd-Mn quasicrystal. The Bergman dodecahedron, which consists of a dodecahedron, an icosahedron and an inner atom (33 atoms in total), does not achieve such an agreement, unless some additional shells are added: alone, it is simply too small. Finally, calculations performed on other combinations of polyhedra or on single-shell polyhedra result in very poor agreement showing the sensitivity of XPD to the variation of the local environment. Therefore, XPD proves to be very useful for independently verifying and completing in a very direct way models proposed via indirect, reciprocal-space based, diffraction methods. 
(a)

\section{Icosidodecahedron}
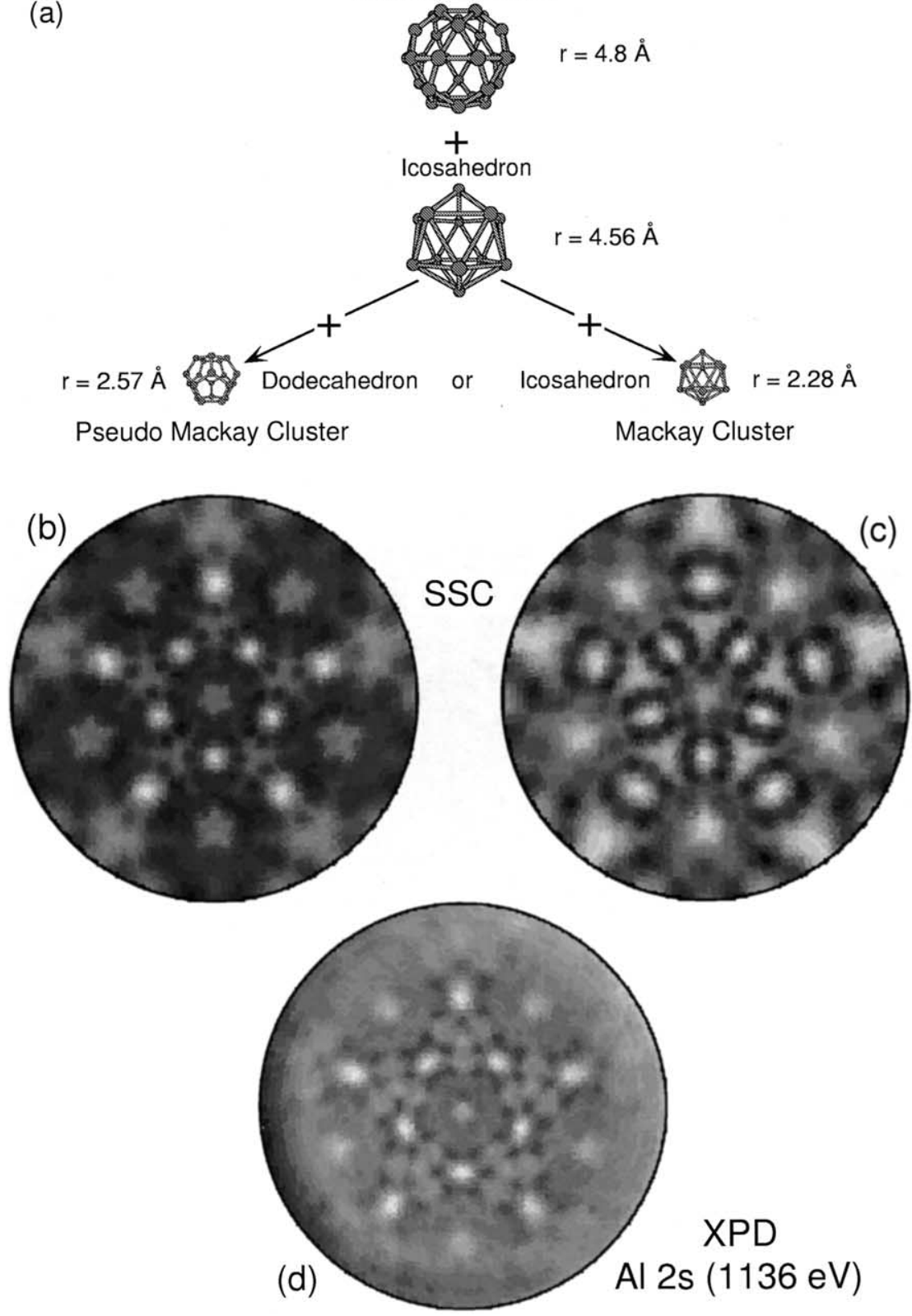

Fig. 7. (a) Description of two clusters built of shells of polyhedra, the two external shells are identical; the inner shell is a dodecahedron (with an occupation of $8 / 20$ ) for the pseudo-Mackay cluster and an icosahedron for the Mackay cluster. Both clusters have an inner central atom (not shown). Simulated SSC maps (A12s emission) using as input atomic positions (b) of a pseudo-Mackay cluster and (c) of a Mackay cluster (d) experimental XPD patterns of a $5 f i$-Al-Pd-Mn surface for $\mathrm{Al}$ emission. 


\section{Low-temperature crystalline phases: structure and orientation}

In this section, we focus on the structure and orientation of ordered crystalline multitwinned overlayers produced by ion bombarding or after annealing the sputtered surface at temperatures below $400{ }^{\circ} \mathrm{C}$ (as shown in Fig. 1). Such reconstructions appear on the three differently oriented surfaces of $i-\mathrm{Al}-\mathrm{Pd}-\mathrm{Mn}$ [12-14], and are also reported by others [33-35]. They are characterised by sharp spots in LEED indicating a different surface ordering than the quasicrystalline icosahedral surface and a drastically modified XPS composition $\left(\mathrm{Al}_{55} \mathrm{Pd}_{45} \mathrm{Mn}_{5}\right)[12,13,33,35]$. The electronic fingerprint of such surfaces is typically metallic with a sharp Fermi cutoff, as discussed in Section 3 and Fig. 3 [13,14].

We decided to show in Fig. 8 the reconstruction of the $5 f$ surface of $i$-Al-Pd-Mn because the reconstructed surface has apparently the geometry of an icosahedral quasicrystalline surface. So, here, we wish to demonstrate how such an interesting feature is mimetised by twinning of crystalline domains on the icosahedral bulk. In Fig. 8a and $\mathrm{b}$ are displayed an experimental Pd diffractogram taken from a $5 f$
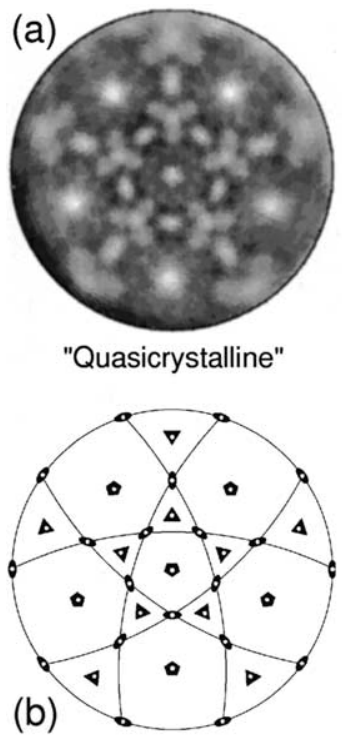

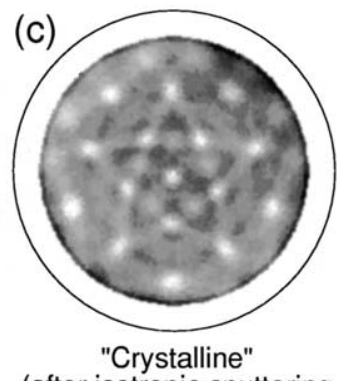

(after isotropic sputtering 5 domains (d) rotated by $72^{\circ}$ )

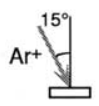

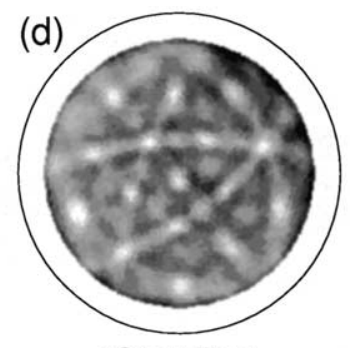

"Crystalline"

(after anisotropic sputtering)

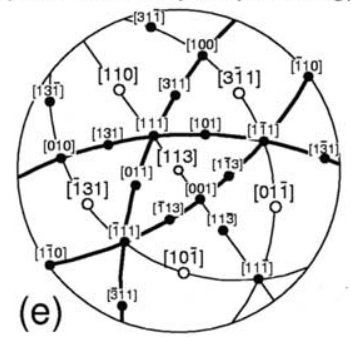

Fig. 8. Low-temperature crystalline phase: (a) experimental XPD patterns of a quasicrystalline surface of $i$-Al-Pd-Mn cut perpendicularly to a $5 f$ symmetry axis $\left(\mathrm{Pd}_{3} \mathrm{~d}_{5 / 2}, E_{\mathrm{kin}}=917 \mathrm{eV}\right)$; (b) stereographic projection of the icosahedral symmetry elements, i.e., axes of $2 f$ (ellipses), $3 f$ (triangles) and $5 f$ (pentagons) symmetry; the lines are linking $2 f$ axes; (c) a reconstruction of the surface appears after isotropic $\mathrm{Ar}^{+}$ sputtering (the sample was rotated during sputtering, same energy as (a)); (d) the development of one of the five possible domains is favoured by anisotropic sputtering the surface (no sample rotation); the reconstruction is identified as a cubic bcc AlPd crystalline alloy oriented along the [1 13] direction; (e) stereographic projection of a cubic bcc(113) structure; (110)-like high-density crystal planes are drawn; the planes emphasized in black are those interconnecting the $2 f$ axes in the icosahedral stereographic projection (b). 
quasicrystalline surface of $i$-Al-Pd-Mn (described in Section 3 and Fig. 4b) and the stereographic projection of the icosahedral symmetry elements (here represented with lines connecting the $2 f$ axes). Fig. $8 \mathrm{c}$ displays the XPD pattern (represented with the same orientation as the bulk) of an isotropically sputtered surface, i.e., as, in our experimental geometry, the impinging sputtering ions reach the surface with an angle of $15^{\circ}$ off normal, we rotate the sample during the sputtering process in order to ensure an homogeneous treatment of the surface. The symmetry of the pattern is still fivefold. However, "Y"-shaped intensity spots (trefoils) which appear in the $3 f$ directions (indicated by triangles in Fig. 8b) of the icosahedral quasicrystalline surface (Fig. 8a) do not exist anymore on the sputtered surface. High-intensity lines, representing high-density planes, link the $2 f$ axes of the icosahedral surface (ellipses in Fig. 8b). Since we were convinced that such features are due to twinning of a crystalline phase at the surface, we tried to favour the development of only one individual domain by inhomogeneously sputtering the surface, i.e., simply without rotating it during the sputtering treatment. The symmetry of the resulting pattern is thus dramatically modified and only a single mirror-symmetry plane starting at the upper left corner remains (Fig. 8d). Two of the five high-density planes present in Fig. $8 \mathrm{c}$ forming the main starlike pattern vanished. This structure can be identified as a single-domain of a cubic bcc structure projected along to its (1113) direction. By comparing the icosahedral surface (Fig. 8a) to the bcc(1 13 ) reconstructed overlayer (Fig. 8d) and by superimposing their respective stereographic projections (Fig. 8b and e), we find striking coincidence between the most intense low-index directions of the two surfaces [14]. Finally, by superimposing five measurements taken from the single-domained bcc(113) overlayer (Fig. 8d) rotated by $72^{\circ}$ with respect to each other, we reconstitute a pentagonal pattern similar to the experiment shown in Fig. 8c. Therefore, the isotropically sputtered surface (Fig. 8c) can be interpreted as five approximately equally populated domains of a cubic bcc AlPd crystalline alloy surface oriented perpendicularly to its [1 113$]$ direction.

In the sputtered $2 f$ and $3 f$ surfaces, we similarly observe the disappearance of the trefoil patterns in the $3 f$ directions, the occurrence of continuous plane lines and coincidences between intense low-index directions. In both cases, the surface consisted of a combination of crystalline bcc domains: for $2 f i$-Al-Pd-Mn, two bcc(1 110$)$ domains rotated by $109^{\circ}$ with respect to each other [13] and, for $3 f i$-Al-Pd-Mn, six bcc(321) domains rotated by $120^{\circ}$. Putting together the results obtained from the three surfaces, one can learn more on the relationship between the two phases.

\section{High-temperature phases (crystalline and decagonal phase) and $\xi^{\prime}-\mathrm{Al}-\mathrm{Pd}-\mathrm{Mn}$ approximant}

In this last section, we characterise overlayers obtained after prolonged annealings of the $5 f i$-Al-Pd-Mn quasicrystal surfaces at temperatures above $650{ }^{\circ} \mathrm{C}$ and we compare their structure and electronic structure data with those taken from a $\xi^{\prime}-\mathrm{Al}-$ Pd-Mn approximant (see Fig. 1). The annealing of sputtered surfaces at high temperatures produces either Pd or Mn enrichment, depending probably on the initial 
bulk composition, but the exact reason is not known. The Pd rich overlayers are crystalline [12,16,35], whereas different terminations were observed for the $\mathrm{Mn}$ enriched surfaces, as a mixture of different secondary phases [21], a crystalline orthorhombic $\mathrm{Al}_{3} \mathrm{Pd}$ surface [20] or, as observed in our group, the stable decagonal $d$-Al-Pd-Mn phase overlayer [18]. In order to recover an icosahedral quasicrystalline surface, treatments as drastic as repolishing [20] or $\sim 5 \mathrm{~h}$ of ion sputtering followed by annealing at about $550{ }^{\circ} \mathrm{C}$ [18] are necessary. The LEED patterns, we observed, on the Pd and $\mathrm{Mn}$ enriched are both $10 f$, but with different features, and, naturally, both are very different from those taken from the icosahedral surface $[12,16,18]$.

Fig. 9 shows experimental diffractograms of Pd emission, presented with the same orientation as the bulk, taken from the quasicrystalline $5 f i$-Al-Pd-Mn surface (see Section 3) and the two possible precipitated phase, the Pd rich and the Mn rich. Note that the different enrichments were not obtained on the same sample. The XPD map

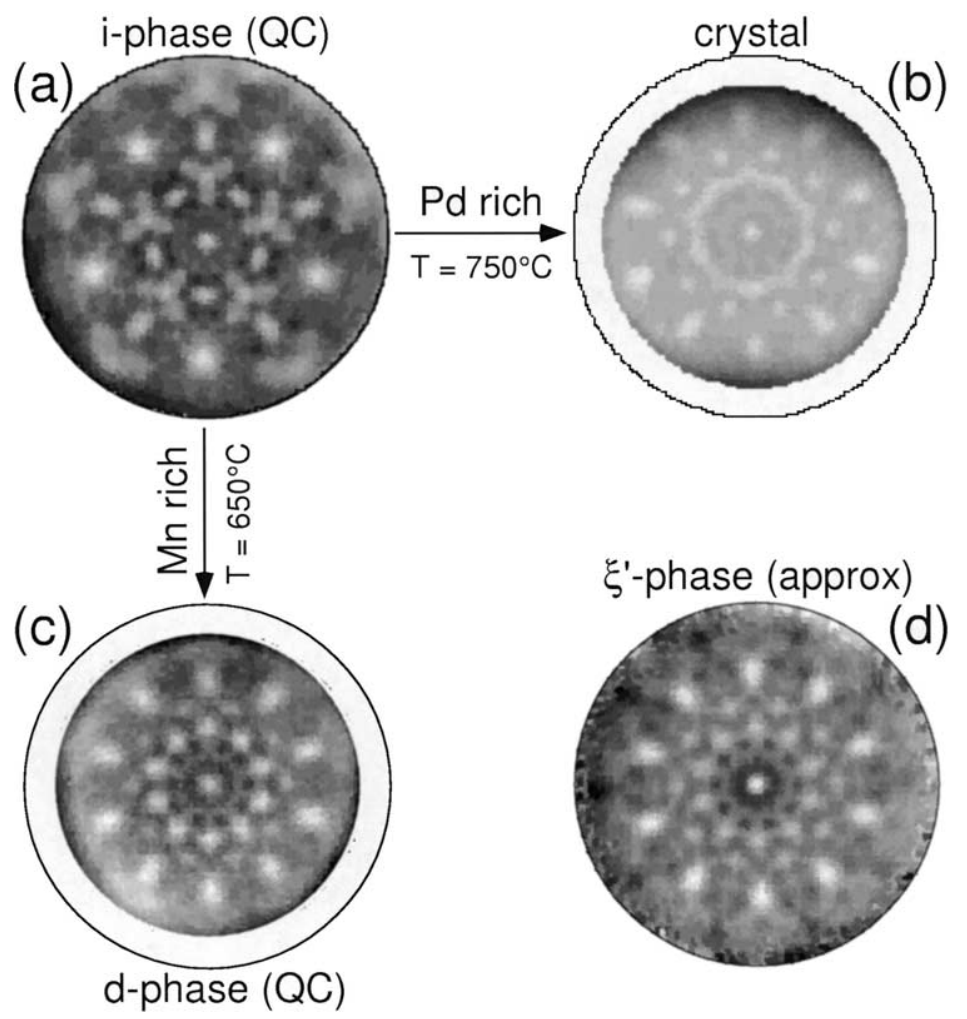

Fig. 9. Two different high-temperature phases (crystalline phase and $d$-phase) and $\xi$-approximant: experimental XPD patterns ( $\mathrm{Pd} 3 \mathrm{~d}_{5 / 2}, E_{\mathrm{kin}}=917 \mathrm{eV}$ ) (a) of a bulk-terminated icosahedral surface of $5 f i$ $\mathrm{Al}-\mathrm{Pd}-\mathrm{Mn}$; (b) of a Pd-rich overlayer formed on $5 f i$-Al-Pd-Mn $\left(T_{\text {anneal }}=750{ }^{\circ} \mathrm{C}\right)$ interpreted as a combination of cubic Al-Pd(1 10$)$ domains; (c) of a Mn-rich overlayer formed on $5 f i$-Al-Pd-Mn $\left(T_{\text {anneal }}=650{ }^{\circ} \mathrm{C}\right)$ identified as the stable decagonal quasicrystal phase; (c) of a bulk-terminated approximant surface (pseudo-10f $\xi^{\prime}$-Al-Pd-Mn). 
of the Pd rich overlayer has a $10 f$ symmetry and the composition is about $\mathrm{Al}_{67} \mathrm{Pd}_{32} \mathrm{Mn}_{<1}$ (Fig. 9b). The central intensity of "Y"-shaped patterns appearing at $3 f$ axes (see stereographic projection Fig. $4 d$ ) disappeared and a continuous ring replaces the alternance of spots visible at $2 f$ and $3 f$ axes in the icosahedral surface. We interpreted this data as a combination of five domains of a cubic Al-Pd(1 110$)$ alloy $[12,16]$. The XPS composition of the Mn rich overlayer ( $\mathrm{Al}_{76} \mathrm{Pd}_{11} \mathrm{Mn}_{13}$, Fig. 9c) is compatible with the composition range of the $d$-phase [19], in contrast to the vastly different composition $\left(\mathrm{Al}_{22} \mathrm{Pd}_{56} \mathrm{Mn}_{22}\right)$ of a metastable decagonal overlayer reported in Ref. [36]. The diffractogram of the Mn rich overlayer is $10 f$ symmetric and, as for the Pd rich overlayer, the trefoil patterns disappeared from $3 f$ axes; but, instead of a continuous ring, here 10 distinct and equivalent spots appear. Additional ex-situ experiments (electron backscatter diffraction (EBSD) by K. Kunze and C. Beeli, ETHZ, Zürich) were performed in order to check the character of the produced layer. From all these measurements (including UPS), we deduced that the overlayer corresponds to the stable decagonal phase [17] and that it is about 200-500 A thick (from the probing depth of EBSD, and from the sputter rate and needed time to remove the layer). As mentioned in Fig. 1, it is possible to form a crystalline surface on the thick $d$-phase overlayer consisting of bcc domains, similarly to what is observed on the $i$-phase surface (Section 5).

The $\xi^{\prime}-\mathrm{Al}_{73.5} \mathrm{Pd}_{22.4} \mathrm{Mn}_{4.1}$ approximant is a crystal with a huge unit cell $(a=23.541$ $\AA, b=16.566 \AA, c=12.339 \AA$ ) and a quasicrystal-like structure within the unit cell [22]. So, this material should exhibit properties close to the quasicrystal. Very recently surface experiments were performed on this material $[33,37]$. The $\mathrm{Pd}$ experimental XPD map of the pseudo- $10 f \xi^{\prime}-\mathrm{Al}-\mathrm{Pd}-\mathrm{Mn}$ approximant is shown in Fig. 9d. The symmetry of the pattern is $10 f$ and the observed features are almost identical to those of the $d$-phase overlayer. This is surprising because it has a different composition (the $\xi^{\prime}$-phase contains much less $\mathrm{Mn}$ ) and a different LEED pattern than the $d$-phase overlayer. It is interesting to note that we do not observe any trace of the periodic character of the approximant in the XPD pattern; but this is likely due to the local short-range character of XPD experiments in comparison with the size of the unit cell.

In order to further characterise these surfaces, in Fig. 10, we show valence-band spectra from a sputtered metallic surface (here a sputtered $2 f \xi^{\prime}$-approximant, which is also bcc crystalline), an approximant pseudo- $10 f \xi^{\prime}-\mathrm{Al}-\mathrm{Pd}-\mathrm{Mn}$ surface, the $d$ phase overlayer formed on $5 f i$-Al-Pd-Mn and the icosahedral $5 f i$-Al-Pd-Mn quasicrystal surface (no data available for the Pd rich phase). As described in Section 3 , the black dots extrapolate the DOS from the measurement (black line) at the nearFermi edge region. The DOS of the crystalline sputtered surface remains high and linear over the complete range of energy and the Fermi edge is sharp as expected for a metal, whereas a distinct decrease of DOS is observed on the icosahedral QC surface (see also Section 3). The density of states of the stable decagonal overlayer (Fig. 10c) is also lowered close to $E_{\mathrm{F}}$. However, the shape of the curve is slightly different, with a steeper Fermi cutoff. This is probably related to the fact that the decagonal surface is periodic in one dimension (along the surface normal). Finally, the DOS of the $\xi^{\prime}$-approximant is also lowered (see Fig. 10b and e), indicating his 


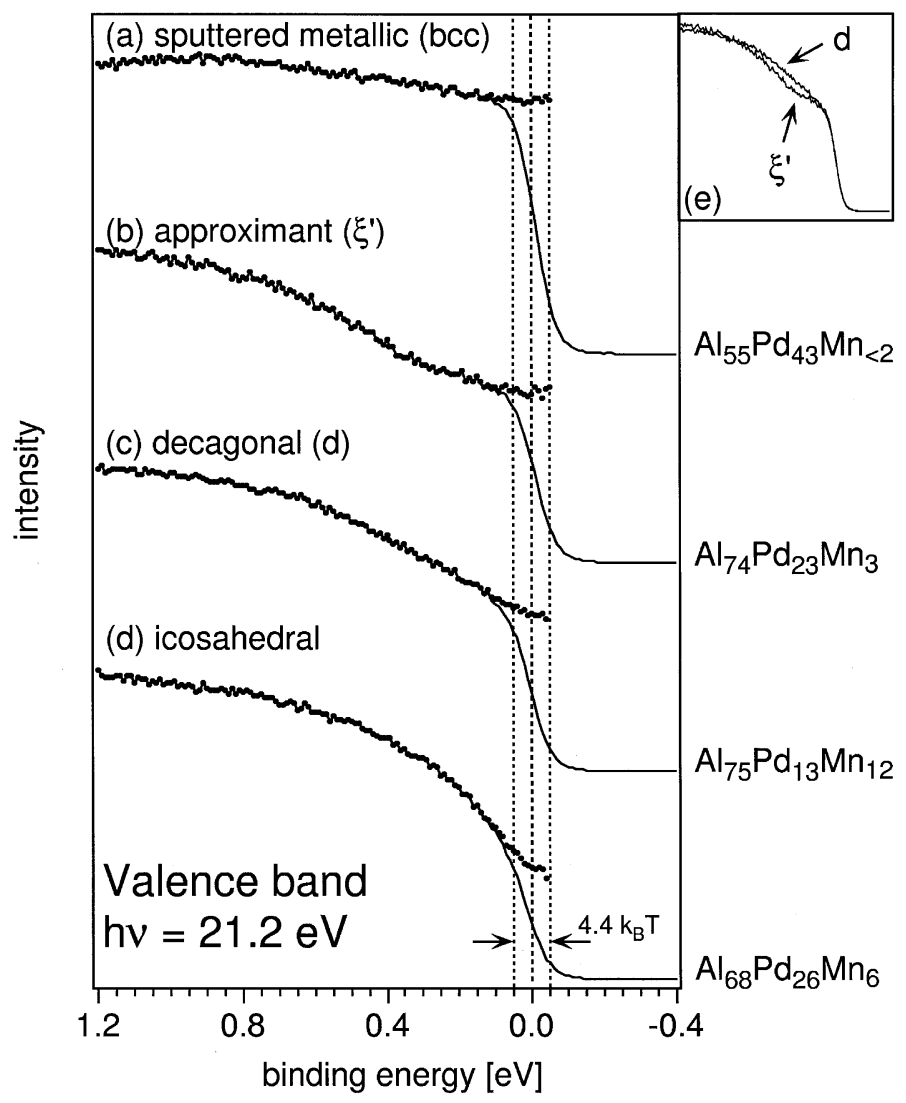

Fig. 10. Valence-band spectra displaying the region near the Fermi edge, taken with monochromatised $\mathrm{He}$ I radiation at room temperature, (a) of a crystalline surface (sputtered $2 f \xi^{\prime}-\mathrm{Al}-\mathrm{Pd}-\mathrm{Mn}$ with a bcc structure); (b) of a bulk-terminated approximant surface (pseudo-10f $\xi^{\prime}-\mathrm{Al}-\mathrm{Pd}-\mathrm{Mn}$ ) (c) of the Mn-rich stable decagonal quasicrystalline overlayer grown on a $5 f$-Al-Pd-Mn quasicrystal, (d) of the bulk-terminated icosahedral quasicrystalline surface ( $5 f i-\mathrm{Al}-\mathrm{Pd}-\mathrm{Mn})$. (e) comparison between the DOS of the $d$-phase quasicrystal (c) and $\xi^{\prime}$-phase approximant (b). XPS compositions are indicated for each spectrum.

intermediate character between a pure metal and the QC (see also the continuous behaviour of the spectral function within the $4.4 k_{\mathrm{B}} T$ ). Nevertheless, experiments at low temperatures are necessary to determine a real metallicity of these surfaces.

\section{Concluding remarks}

The structure and electronic structure of different low-index surfaces of either quasicrystalline $i-\mathrm{Al}-\mathrm{Pd}-\mathrm{Mn}$ or approximant $\xi^{\prime}-\mathrm{Al}-\mathrm{Pd}-\mathrm{Mn}$ were studied. XPD is a 
powerful chemically selective technique, providing at the same time local, site-specific, real-space information on the structure of the near-surface region. We found that the $2 f, 3 f$ and $5 f$ surfaces of $i-\mathrm{Al}-\mathrm{Pd}-\mathrm{Mn}$ exhibit all the symmetry elements of the icosahedral non-crystallographic group within the probed depth of approximately 20-50 A (depending on the energy of photoelectrons). These XPD experiments were modeled by SSC calculations and are in agreement with the bulk structure.

As the experiments are performed in UHV, the samples are cleaned in situ by sputter/anneal cycles, which induce composition changes by preferential ion sputtering and by thermal diffusion with annealing. The bulk-like terminated icosahedral or approximant surfaces are restored after annealing at about $500-600{ }^{\circ} \mathrm{C}$. For $T<400^{\circ} \mathrm{C}$, ordered crystalline surfaces with bcc domains, mimetising the symmetry of the underlying bulk, are formed, whereas annealing at $T>600{ }^{\circ} \mathrm{C}$ results in $\mathrm{Mn}$ or $\mathrm{Pd}$ enrichment of the surface corresponding to the formation of a decagonal quasicrystalline overlayer or a crystalline layer with bcc domains, respectively. The produced phases exhibit interesting epitaxial coincidences with the axes of the underlying bulk, but are characterised by a loss of intensity maxima in the $3 f$ directions (trefoils) for $i$-Al-Pd-Mn. However, due to the local short-range character of XPD experiments, it is difficult to distinguish between the pseudo- $10 f$ surfaces of an $\xi^{\prime}-\mathrm{Al}-\mathrm{Pd}-\mathrm{Mn}$ approximant and $10 \mathrm{f}$ face of a decagonal quasicrystal.

The (low-temperature) crystalline overlayers are characterised by a sharp Fermi edge and a linear DOS, while the icosahedral and decagonal QC surfaces and the approximant surface have a lowered DOS close to $E_{\mathrm{F}}$ but with a different shape. The DOS of the icosahedral QC surface is compatible with the opening of a pseudogap, as expected for a quasicrystal. The decagonal QC and approximant surface, as they are periodic in one direction or crystalline containing a QC-like structure within its large unit cell, respectively, exhibit an intermediate character between the typical quasicrystal and a purely metallic surface.

\section{Acknowledgements}

I am particularly grateful to Y. Calvayrac, S. Lefebvre and M. Bessière for providing me the very first quasicrystal samples in summer 1994 during my postdoc stay at LURE, Orsay and to H.-U. Nissen for discussing the very first results during the same summer. I wish to thank the persons who provided many further excellent quality monogram quasicrystal samples (T.A. Lograsso, D.W. Delaney, A.R. Ross, $\mathrm{Ph}$. Ebert) as well as my closest collaborators on this project (P. Aebi, C. Beeli, K. Kunze, L. Schlapbach). I also acknowledge the members and ex-members of the XPD team (now located at the University of Neuchâtel) as well as the technical staff, and also many other persons of the QC community (among them M. Boudard, J.-M. Dubois, C. Janot, C.J. Jenks, P.A. Thiel) for help and stimulating discussions. This project has been supported by the Fonds National Suisse de la Recherche Scientifique and the "Smart Quasicrystal" EU Project (GRD-2001-30054 and BBW No. 00.0508-1 for Switzerland). 


\section{References}

[1] D. Shechtman, I. Blech, D. Gratias, J.W. Cahn, Phys. Rev. Lett. 53 (1984) 1951.

[2] C. Janot, Quasicrystals: A Primer, 2nd ed., Oxford University Press, Oxford, 1994;

Z.M. Stadnik (Ed.), Physical Properties of Quasicrystals, Springer Series in Solid-State Sciences 126, Berlin, 1999.

[3] T.M. Schaub, D.E. Bürgler, H.-J. Güntherodt, J.-B. Suck, Phys. Rev. Lett. 73 (1994) 1255.

[4] M. Erbudak, H.-U. Nissen, E. Wetli, M. Hochstrasser, S. Ritsch, Phys. Rev. Lett. 72 (1994) 3037.

[5] T.M. Schaub, D.E. Bürgler, H.-J. Güntherodt, J.-B. Suck, Z. Phys. B 96 (1994) 93.

[6] For a review see, e.g., C.S. Fadley, Surf. Sci. Rep. 19 (1993) 231.

[7] S.J. Poon, Adv. Phys. 41 (1992) 303.

[8] G. Neuhold, S.R. Barman, K. Horn, W. Theis, Ph. Ebert, K. Urban, Phys. Rev. B 58 (1998) 734.

[9] X. Wu, S.W. Kycia, C.G. Olson, P.J. Benning, A.I. Goldman, D.W. Lynch, Phys. Rev. Lett. 75 (1995) 4540.

[10] E. Rotenberg, W. Theis, K. Horn, P. Gille, Nature 406 (2000) 602.

[11] A.P. Tsai, Y. Yokoyama, A. Inoue, T. Masumoto, Philos. Magn. Lett. 61 (1990) 9.

[12] D. Naumović, P. Aebi, L. Schlapbach, C. Beeli, T.A. Lograsso, D.W. Delaney, in: S. Takeuchi, T. Fujiwara (Eds.), Proceedings of the 6th International on Quasicrystals (ICQ-6, Yamada Conference XLVII), World Scientific, Singapore, 1998, p. 749.

[13] D. Naumović, P. Aebi, L. Schlapbach, C. Beeli, T.A. Lograsso, D.W. Delaney, Phys. Rev. B 60 (1999) R16330.

[14] D. Naumović, P. Aebi, L. Schlapbach, C. Beeli, Mat. Sci. Eng. A 294-296 (2000) 882.

[15] D. Naumović, P. Aebi, L. Schlapbach, C. Beeli, in: A.I. Goldman, D.J. Sordelet, P.A. Thiel, J.M. Dubois (Eds.), New Horizons in Quasicrystals, Research and Applications, World Scientific, Singapore, 1997 , p. 86.

[16] D. Naumović, P. Aebi, C. Beeli, L. Schlapbach, Surf. Sci. 433-435 (1999) 302.

[17] C. Beeli, H.-U. Nissen, J. Robadey, Philos. Magn. Lett. 63 (1991) 87.

[18] D. Naumović, P. Aebi, L. Schlapbach, C. Beeli, K. Kunze, T.A. Lograsso, D.W. Delaney, Phys. Rev. Lett. 87 (2001) 195506.

[19] T. Gödecke, R. Lück, Z. Metallk. 86 (1995) 109.

[20] J. Ledieu, C.A. Muryn, G. Thornton, G. Cappello, J. Chevrier, R.D. Diehl, T.A. Lograsso, D.W. Delaney, R. McGrath, Mat. Sci. Eng. A 294-296 (2000) 871.

[21] P.A. Thiel, in: Z.M. Stadnik (Ed.), Physical Properties of Quasicrystals, Springer Series in Solid-State Sciences 126, Berlin, 1999, p. 327.

[22] M. Boudard, H. Klein, M. de Boissieu, M. Audier, H. Vincent, Philos. Magn. A 74 (1996) 939.

[23] D. Popović, D. Naumović, M. Bovet, C. Koitzsch, L. Schlapbach, P. Aebi, Surf. Sci. 492 (2001) 294.

[24] J. Osterwalder, T. Greber, A. Stuck, L. Schlapbach, Phys. Rev. B 44 (1991) 13764.

[25] D. Naumović, A. Stuck, T. Greber, J. Osterwalder, L. Schlapbach, Phys. Rev. B 47 (1993) 7462.

[26] T. Schaub, J. Delahaye, C. Berger, H. Guyot, R. Belkhou, A. Taleb-Ibrahimi, Y. Calvayrac, Eur. Phys. J. B 20 (2001) 183.

[27] Z.M. Stadnik, D. Purdie, M. Garnier, Y. Baer, A.-P. Tsai, A. Inoue, K. Edagawa, S. Takeuchi, Phys. Rev. Lett. 77 (1996) 1777.

[28] M. Boudard, M. de Boissieu, C. Janot, G. Heger, C. Beeli, H.-U. Nissen, H. Vincent, R. Ibberson, M. Audier, J.-M. Dubois, J. Phys. Condens. Matter 4 (1992) 10149;

M. de Boissieu, P. Guyot, M. Audier, in: F. Hippert, D. Gratias (Eds.), Lectures on Quasicrystals, Les Editions de Physique, Les Ulis, 1994, pp. 1-152.

[29] C. Janot, M. de Boissieu, Phys. Rev. Lett. 72 (1994) 1674;

C. Janot, Phys. Rev. B 53 (1996) 181.

[30] A. Katz, D. Gratias, J. Non-Cryst. Solids 153-154 (1993) 187;

A. Katz, D. Gratias, in: C. Janot, R. Mosseri (Eds.), Proc. of the 5th Int. Conf. on Quasicrystals, World Scientific, Singapore, 1995, p. 164.

[31] V. Elser, Philos. Magn. B 73 (1996) 641. 
[32] M. Gierer, M.A. Van Hove, A.I. Goldman, Z. Shen, S.-L. Chang, C.J. Jenks, C.-M. Zhang, P.A. Thiel, Phys. Rev. Lett. 78 (1997) 467.

[33] Z. Shen et al., Phys. Rev. Lett. 78 (1997) 1050;

Phys. Rev. B 58 (1998) 9961;

Surf. Sci. 450 (2000) 1.

[34] B. Bolliger, M. Erbudak, D.D. Vvedensky, M. Zurkirch, A.R. Kortan, Phys. Rev. Lett. 80 (1998) 5369 ;

B. Bolliger, M. Erbudak, A. Hensch, D.D. Vvedensky, Mat. Sci. Eng. A. 294-296 (2000) 859.

[35] F. Schmithüsen, G. Cappello, M. de Boissieu, M. Boudard, F. Comin, J. Chevrier, Surf. Sci. 444 (2000) 113.

[36] B. Bolliger, M. Erbudak, D.D. Vvedensky, A.R. Kortan, Phys. Rev. Lett. 82 (1999) 763.

[37] V. Fournée, P.J. Pinhero, J.W. Anderegg, T.A. Lograsso, A.R. Ross, P.C. Canfield, I.R. Fisher, P.A. Thiel, Phys. Rev. B 62 (2000) 10049. 